\title{
Bypassing multidrug resistance in human breast cancer cells with lipid/polymer particle assemblies
}

This article was published in the following Dove Press journal:

International Journal of Nanomedicine

6 January 2012

Number of times this article has been viewed

Bo $\mathrm{Li}^{\prime}$

Hui $X u^{2}$

Zhen $\mathrm{Li}^{\prime}$

Mingfei Yao'

Meng Xie'

Haijun Shen'

Song Shen'

Xinshi Wang'

Yi Jin'

'College of Pharmaceutical sciences,

Zhejiang University, Hangzhou,

${ }^{2}$ No. 202 Hospital of People's

Liberation Army, Shenyang, China
Correspondence: $Y i$ Jin

866 Yuhangtang Road, College

of Pharmaceutical Sciences, Zhejiang

University, Hangzhou 310058 China

Tel +8657188208435

Fax +86 57। 88208435

Email jinyizju@hotmail.com
Background: Multidrug resistance (MDR) mediated by the overexpression of adenosine triphosphate (ATP)-binding cassette $(\mathrm{ABC})$ transporters, such as P-glycoprotein (P-gp), remains one of the major obstacles to effective cancer chemotherapy. In this study, lipid/ particle assemblies named LipoParticles (LNPs), consisting of a dimethyldidodecylammonium bromide (DMAB)-modified poly(lactic-co-glycolic acid) (PLGA) nanoparticle core surrounded by a 1,2-dipalmitoyl-sn-glycero-3-phosphocholine (DPPC) shell, were specially designed for anticancer drugs to bypass MDR in human breast cancer cells that overexpress P-gp.

Methods: Doxorubicin (DOX), a chemotherapy drug that is a P-gp substrate, was conjugated to PLGA and encapsulated in the self-assembled LNP structure. Physiochemical properties of the DOX-loaded LNPs were characterized in vitro. Cellular uptake, intracellular accumulation, and cytotoxicity were compared in parental Michigan Cancer Foundation (MCF)-7 cells and P-gp-overexpressing, resistant MCF-7/adriamycin (MCF-7/ADR) cells.

Results: This study found that the DOX formulated in LNPs showed a significantly increased accumulation in the nuclei of drug-resistant cells relative to the free drug, indicating that LNPs could alter intracellular traffic and bypass drug efflux. The cytotoxicity of DOX loaded-LNPs had a 30 -fold lower half maximal inhibitory concentration $\left(\mathrm{IC}_{50}\right)$ value than free $\mathrm{DOX}$ in MCF-7/ADR, measured by the colorimetric cell viability (MTT) assay, correlated with the strong nuclear retention of the drug.

Conclusion: The results show that this core-shell lipid/particle structure could be a promising strategy to bypass MDR.

Keywords: chemotherapy, drug delivery, polymeric nanoparticles, multidrug resistance

\section{Introduction}

The development of multidrug resistance (MDR) is a major obstacle to effective cancer chemotherapy. After a long period of chemotherapy, many patients suffer from MDR, which can reduce therapy efficiency and lead to treatment failure. ${ }^{1}$ The high level of resistance is usually caused by complex MDR mechanisms. Among them, overexpression of the adenosine triphosphate (ATP)-binding cassette transporters (ABC), such as P-glycoprotein (P-gp), is one of the most prevalent mechanisms., ${ }^{2,3}$ Tumor cells possess highly ordered internal resistance. This P-gp encoded by the MDR-1 gene acts as a drug efflux pump that exports a wide range of chemotherapeutic drugs and will reduce the accumulation of functional drugs in MDR cancer cells, resulting in low cancer chemotherapeutic efficacy. ${ }^{4}$ Currently, only a few tissuederived advanced cancers can be cured by chemotherapeutic drugs. Furthermore, these tumors may also recur and become drug resistant. ${ }^{5}$ Therefore, overcoming the 
drug resistance of tumors is a serious challenge for successful chemotherapy.

With the development of nanotechnology, nanoformulations have been widely used to circumvent MDR. ${ }^{6,7}$ Previous studies have shown that these nano-sized particles, such as lipids, micelles, and inorganic hybrid particles, can bypass the P-gp efflux pumps and alter the intracellular accumulation of chemotherapeutic drugs. ${ }^{8,9}$ In a recent study, biodegradable polymer capsules offered a promising means to circumvent P-gp-mediated MDR. ${ }^{10}$ The capsules were internalized by MDR cells via endocytosis, and were designed for the subsequent intracellular release of the drugs by enzymatic degradation in lysosomes. According to another strategy, low molecular weight polyethylenimine (PEI) conjugated to doxorubicin (DOX)-loaded particles could escape endosomal retention, and increase the intracellular DOX accumulation, thereby circumventing MDR-mediated drug efflux. ${ }^{11}$ These synthetic biomaterials are able to escape the endosomal/ lysosomal compartment and change intracellular trafficking to increase the drug concentration in MDR cancer cells. These are common mechanisms whereby MDR activity can be reversed. Thus, various endosomal escape pathways provide an opportunity for encapsulating drugs to overcome MDR in tumors. ${ }^{12}$

Several synthetic agents that have been introduced, such as PEI and poly(amidoamine)s (PAAs), have the proton sponge effect, a property that can contribute to efficient endosomal escape. ${ }^{13,14}$ In this study, dimethyldidodecylammonium bromide (DMAB), which is a cationic emulsifier with double hydrocarbon chains, was used to escape the endosomal pathway. Early nanotechnology application of DMAB focused on oral drug delivery or inhibition of vascular restenosis because of its low critical micelle concentration and high zeta potential. ${ }^{15,16}$ Recent studies have shown that DMAB-modified nanoparticles are an ideal vehicle for nuclear targeting that could significantly improve the transfection rate in gene delivery. ${ }^{17}$ The results demonstrated clearly that DMAB-modified nanoparticles could evade the arrest of the endosomal/lysosomal lumen and destabilize the membranes. This is similar to the effects elicited by the lysosomal disrupting agent L-Leucine-L-leucine methyl ester (LLME). ${ }^{18}$ The carrier then escapes from the endosomes/ lysosomes and is targeted to the cell nucleus. These and other studies with drug- or gene-loaded DMAB nanoparticles have demonstrated their advantage as efficient drug delivery vehicles with small particles sizes, enhanced cell uptake, and endosomal escape. ${ }^{19,20}$ However, no studies have been carried out to improve the biocompatibility of DMAB particles for in vivo applications, or to examine whether drug-loaded DMAB particles are able to circumvent MDR by efficiently escaping endosomes and lysosomes.

In recent years, the core-shell supramolecular structures, the lipid/particle assemblies - termed LipoParticles (LNPs) have received considerable attention for their potential application in biomedicine and biotechnology. ${ }^{21,22}$ These LipoParticles assemblies, based on electrostatic interaction, were first reported by Carmona-Ribeiro et al. ${ }^{23,24}$ Later, Troutier et al reported an in-depth characterization on the basis of zeta potential measurements and microscopic morphology. ${ }^{25,26}$ Subsequent studies demonstrated that these unique hybrid assemblies, consisting of a solid polymer sphere surrounded by a lipid shell, could combine the advantages of lipid shells with those of solid support cores. ${ }^{27}$ The lipid shell provides excellent biocompatibility, as well as the ability to encapsulate particles for in vitro and in vivo bioapplications for protein, peptide, and DNA (lipidic liposome/DNA complexes) delivery. ${ }^{28}$ Lipid/particle assemblies, based on cationic polysaccharide nanoparticles as oligonucleotide carriers, appear to be endocytosed and accumulate intracellularly in the cytoplasm, further indicating additional structural advantages. ${ }^{29}$

The current study designed a novel, core-shell structure lipid/particle assembly, LNPs, based on the cationic DMABmodified poly(lactic-co-glycolic acid) (PLGA) nanoparticles to overcome MDR. The PLGA nanoparticle core was wrapped by 1,2-dipalmitoyl-sn-glycero-3-phosphocholine (DPPC) via hydrophilic and electrostatic interactions and forming a stable, cell-like structure. ${ }^{30}$ Moreover, the phospholipid surface was expected to improve biocompatibility and enhance the penetration of barriers. DOX, a cell cycle toxic drug, was conjugated to PLGA, as reported previously. ${ }^{31}$ In this manner, DOX was loaded in a stable manner, and the drug release could also be controlled. The core-shell LNP utilizes DMAB-modified DOX-PLGA nanoparticles as a strategy to escape the endosomal/lysosomal pathway by enzymatic degradation to overcome MDR in cancer cells. ${ }^{10}$ The mechanism for reversing MDR was confirmed by intracellular accumulation observations.

\section{Materials and methods Materials}

PLGA block copolymer (50:50, molecular weight: 8000) was purchased from Shandong Institute of Medical Instruments (Jinan, China). DPPC was purchased from Avanti Polar Lipids Inc (Alabaster, AL). DOX was purchased from Haikou Manfangyuan Chemical Company (Haikou, China). DMAB, 
Trinton ${ }^{\circledR}$ X-100, and thiazolyl blue tetrazolium bromide was obtained from Sigma-Aldrich ${ }^{\circledR}$ Chemical Co, (St Louis, MI). Ethyl acetate (EA, analytical grade) and acetonitrile (high-performance liquid chromatography (HPLC) grade) was from Merck (Darmstadt, Germany). Distilled water, Milli- ${ }^{\circledR}$ (18 M $\Omega$; Millipore, Billerica, MA), was used in the synthesis. All other materials were analytical grade. Fetal bovine serum (FBS), RPMI-1640 medium (RPMI), penicillin-streptomycin (PS, $10000 \mathrm{U} / \mathrm{mL}$ ), trypsin-EDTA solution ( $0.5 \%$ trypsin, 5.3 Mm EDTA tetrasodium), and Hank's balanced salt solution (HBSS) were obtained from Gibco $^{\circledR}$ BRL (Gaithersberg, MD).

\section{Preparation of LNPs}

DOX-PLGA conjugate was synthesized as reported, and was used to prepare nanoparticles. Nanoparticles were prepared by the emulsification-diffusion method. In detail, $200 \mathrm{mg}$ PLGA-DOX conjugate was dissolved in $10 \mathrm{~mL}$ ethyl acetate. Then, the oil phase was slowly added into $20 \mathrm{~mL}$ of an aqueous solution containing 0.5\% DMAB, and emulsified using a high speed homogenizer (FLUKO, Shanghai, China) twice at $10,000 \mathrm{r} / \mathrm{min}$ for 10 minutes, 5 minutes each time. The resulting oil-in-water emulsion solution was slowly poured into $100 \mathrm{~mL}$ water under the moderate magnetic stirring. The organic solvents were removed through rotary-evaporation. The formed nanoparticles were collected by centrifugation (J2-MC; Beckman Coulter, Fullerton, CA; 51,000 g, 30 minutes, $4^{\circ} \mathrm{C}$ ) and washed with distilled water for three times. The nanoparticle pellet was resuspended in solution for further study. Blank nanoparticles were also prepared by the same method. DPPC was dissolved in chloroform; the solution was incubated at $40^{\circ} \mathrm{C}$ for 4 hours with backflow device; then chloroform was removed by rotary evaporation. A suspension of DMAB-modified nanoparticles was added to the DPPC lipid membranes. After stirring and 30 minutes incubation at $45^{\circ} \mathrm{C}$, the suspension was homogeneous with Nuclepore $^{\mathrm{TM}} 100 \mathrm{~nm}$ (Whatman ${ }^{\circledR}$, Brentford, UK). The suspension was centrifuged $\left(16,000 \mathrm{~g}, 30\right.$ minutes, $\left.4^{\circ} \mathrm{C}\right)$ and washed with distilled water three times. The pellet was resuspended in solution or freeze-dried for further study.

\section{Nanoparticle characterization}

\section{Particle size and size distribution}

Mean hydrodynamic diameters and mean size distributions were determined at $25^{\circ} \mathrm{C}$ by means of dynamic-light scattering technique with particle size analyzer (Zetasizer Nano S90; Malvern Instruments, Malvern, UK). The measurement angle was $90^{\circ}$. The nanoparticle samples were freeze-dried and dispersed in a $1 \mathrm{mmol} \cdot \mathrm{L}^{-1} \mathrm{NaCl}$ solution or serumsupplemented RPMI-1640, and the results was given by the average of three measurements. The polydispersity index (PDI) was a dimensionless measure of size distribution, and was also recorded at this stage.

\section{Surface charge}

Zeta potential is an indicator of surface charge, which determines particle stability in dispersion. Zeta potential was performed on a zeta potential analyzer (90Plus; Brookhaven Instrument Corp, Huntsville, NY) by the same treatment as size measurement.

\section{Transmission electron microscopy}

Transmission electron microscopy (TEM) images were performed on a JEM-1230 electron microscope (JEOL, Tokyo, Japan) at an acceleration voltage of $80 \mathrm{kV}$. To prepare sample sections of bare particles or polymeric nanoparticles, the samples were diluted with phosphate-buffered saline (PBS) (NaOH $\left.29 \mathrm{mM}, \mathrm{KH}_{2} \mathrm{PO}_{4}, 50 \mathrm{mM}, \mathrm{pH} 7.0\right)$ and the final particle concentration was $1 \mathrm{mg} / \mathrm{mL}$. The resulting sample was dropped on to a copper grid. Excess aqueous solution was blotted with filter paper and evaporated at room temperature for 2 hours before observation.

\section{Scanning electron microscopy}

Morphology of the nanoparticles was performed on a field emission-scanning electron microscopy (FE-SEM) (S-4800 UHR; Hitachi High Technologies, Tokyo, Japan). The sample was freeze-dried and fixed on metallic studs with double-sided conductive tape. It was then precoated with platinum before SEM observation.

\section{Loading efficiency of nanoparticles}

Untrapped drugs were removed by centrifugation. The nanoparticle samples were freeze-dried. Then 3 or $4 \mathrm{~mL}$ of nanoparticles or polymeric nanoparticles were dissolved in $10 \mathrm{~mL}$ dimethylformamide (DMF) solution. The drug concentration was determined by HPLC (Agilent 1100; Hewlett Packard HP/Agilent Technologies, Santa Clara, CA). The drug loading content (LC) was calculated.

\section{Drug release}

DOX-loaded polymeric nanoparticles $5 \mathrm{~mL}$ were diluted into $1 \mathrm{~mL}$ 1/15 M PBS (pH 5.5, 6.5, and 7.4), in a dialysis bag of MW 3500 (Boya Biotechnology, Shanghai, China). The dialysis bags containing samples were placed into $20 \mathrm{~mL}$ PBS, $37^{\circ} \mathrm{C}$ incubator shaker at $150 \mathrm{r} / \mathrm{min}$. At appropriate intervals, 
5.0 mL solution was removed for determination by HPLC, and fresh release medium was replaced immediately. Percent drug released from polymeric nanoparticles was calculated.

\section{P-gp expression}

MCF-7 cells and MCF-7/ADR cells were allowed to grow in $25 \mathrm{~mL}$ cell bottles separately for 72 hours. One million cells were collected for each sample and were centrifuged at $4000 \mathrm{r} / \mathrm{min}$ for 3-4 minutes. After washing each sample three times by bovine serum albumin (BSA) $(0.1 \mathrm{mg} / \mathrm{mL})$, P-gp specific primary antibody (Abcam, Cambridge, UK) were added and incubated for 1 hour. Then cells were washed another three times by BSA $(0.1 \mathrm{mg} / \mathrm{mL})$ and fluorescein isothiocyanate-labeled secondary antibody (MultiSciences Biotech Co, Ltd, Beijing, China) was added. Again 1 hour later, cells were washed twice by PBS ( $\mathrm{pH} 7.4)$ and analyzed using flow cytometry (FACS) for the green fluorescence intensity. Untreated cells were taken as controls for both cells.

\section{Cellular uptake assay}

MCF-7 parental cells and resistant MCF-7/ADR cells were obtained from the Institute of Biochemistry and Cell Biology of Chinese Academy of Sciences (IBCB, Shanghai, China). Cells were cultured in RPMI-1640 medium with $1 \%$ penicillinstreptomycin solution, supplemented with $10 \% \mathrm{FBS}$ at $37^{\circ} \mathrm{C}$, $5 \% \mathrm{CO}_{2}$, and $95 \%$ humidity. In the present study, $5 \times 10^{4}$ cells were seeded on 24 well black plates (Costar, Acton, MA), which were incubated until the cells reached $80 \%$ confluence. The medium was changed with Hank's HBSS (pH 7.4) and incubated for 30 minutes. After equilibration, the cells were incubated with nanoparticle suspension $(8 \mu \mathrm{g} / \mathrm{mL}$ free DOX or a concentration of LNPs-DOX equivalent to $8 \mu \mathrm{g} / \mathrm{mL}$ in HBSS). The samples were incubated with the cells for $0.50-4$ hours at $37^{\circ} \mathrm{C}$. Then, the cells were centrifuged and washed using PBS ( $\mathrm{pH} 7.4)$ to eliminate free particles that were not preyed by the cells. After washing three times, FACS analysis was used to determine DOX intensity. Negative control was also determined for regulating the results.

\section{Confocal laser scanning microscopy}

Cells were seeded on glass-bottom culture dishes and incubated at $37^{\circ} \mathrm{C}, 5 \% \mathrm{CO}_{2}$, and $95 \%$ relative humidity. After $80 \%$ confluent, the medium was removed and replaced by HBSS. Thirty minutes later, the solution was replaced with free DOX, or LNPs-DOX with the same DOX concentration. The cells were incubated with particles at $37^{\circ} \mathrm{C}$ for 4 hours. Then the cells were washed three times with PBS ( $\mathrm{pH} 7.4)$ solution to eliminate free particles that were not removed by cell uptake. Cells were then fixed with $4 \%$ paraformaldehyde in PBS for 30 minutes, and nuclei were stained by Hoechst 33342 (Beyotime Biotechnology, Nanjing, China). The samples were examined with the confocal laser scanning microscope (LSM 510; Zeiss, Dresden, Germany). The excitation wavelengths were $405 \mathrm{~nm}$ and $488 \mathrm{~nm}$ (Hoechst, blue; DOX, red), respectively.

\section{Dose-response experiments}

MCF-7 parental cells and resistant MCF-7/ADR cells were seeded in 96 well plates at a density of $1 \times 10^{4}$ cells per well and incubated for 24 hours. After that, the medium was replaced with the specified samples. The cells were then treated with free DOX or polymeric nanoparticles at a certain concentration of DOX in $150 \mu \mathrm{L}$ RPMI-1640 medium. Cell viability was determined after 24-hour incubation. Thiazolyl blue tetrazolium bromide solution was added into each well. Without any additions, the cells were incubated at $37^{\circ} \mathrm{C}$ and $5 \% \mathrm{CO}_{2}$ for 4 hours. Subsequently, the culture medium in each well was removed, and $200 \mu \mathrm{L}$ dimethyl sulfoxide (DMSO) was used to dissolve the formed formazan crystals. The absorbance was measured at $570 \mathrm{~nm}$ after shaking using an enzyme-linked immunosorbent assay (ELISA) plate reader (Thermo Scientific Multiskan ${ }^{\circledR}$ Spectrum; Thermo Scientific, Waltham, MA). Cells incubated with fresh RPMI1640 medium were used as a control.

\section{Statistical analysis}

Statistical analysis was performed using t-test. Differences between groups were designated as $P<0.05(*)$.

\section{Results}

\section{Preparation and characterization of LNPs}

In this study, the LNPs were designed for reversing MDR with DMAB-modified PLGA nanoparticle (NP) core coated with DPPC (Figure 1). DOX and PLGA were connected via an amide bond, as reported (supporting information, S1). ${ }^{31}$ The DOX conjugation efficiency was $46 \%$ measured by nuclear magnetic resonance. This kind of DOX-PLGA conjugate could facilitate zero-order release of DOX at the tumor site for a long period. PLGA NPs prepared by DMAB dissolved in ethyl acetate had a uniform size distribution of about $150 \mathrm{~nm}$. The LNPs were self-assembled from the cationic DMABmodified PLGA NP core. The PLGA NPs were wrapped in DPPC via electrostatic forces, and the size of the formed LNPs increased to approximately $200 \mathrm{~m}$. The DOX loading content of the PLGA NPs and LNPs was $0.71 \% \pm 0.06 \%$ and $0.52 \% \pm$ $0.10 \%$, respectively, as measured by HPLC (Table 1 ). 


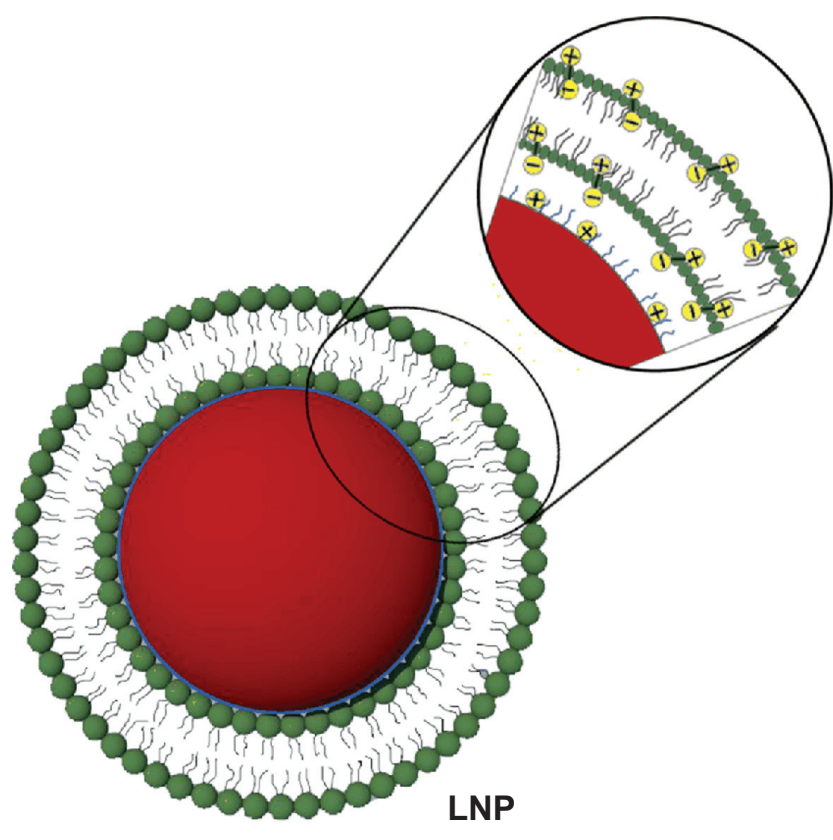

Figure I Schematic diagram showing the structure of lipid/polymer particle assemblies. The negative phosphate charges on the hydrophilic segment of DPPC (green) can be attracted to the oppositely charged ions of the ammonium bromide ammonia headgroup of DMAB (blue), which modify the surface of DOX-loaded PLGA nanoparticles (red). Abbreviations: DMAB, dimethyldidodecylammonium bromide; DOX, doxorubicin DPPC, I,2-dipalmitoyl-sn-glycero-3-phosphocholine; LNP, lipid/polymer particle assembly; PLGA, poly(lactic-co-glycolic) acid.

The surface morphology of LNPs was studied by means of TEM and SEM (Figure 2). Based on TEM images (Figure 2A and B), the LNPs showed relatively uniform sizes and were surrounded by a layer of petal-like vesicles with a bilayer thickness of about $20 \mathrm{~nm}$. SEM images (Figure 2C and D) showed that the surface structure of the PLGA NPs modified by DMAB was fairly rigid, and did not show the decomposition under the effect of electron-beam bombardment. The surface of the LNPs showed evidence of degradation and cracking under the same voltage conditions. These results demonstrate that the nanoparticles were covered by a layer of DPPC. ${ }^{32}$ PLGA NPs had a high positive zeta potential of $57 \mathrm{mV}$ owing to the cationic DMAB. The surface properties of the PLGA core should be changed with the DPPC coating, and the zeta potential of LNPs was reduced from $57 \mathrm{mV}$ to $-1.8 \mathrm{mV}$ (Table 1 ). This negative zeta potential

Table I Physicochemical properties of different formulations $(\mathrm{n}=3)$

\begin{tabular}{lllll}
\hline Sample & Size $(\mathbf{n m})$ & ZP $(\mathbf{m V})$ & PDI & LC (\%) \\
\hline PLGA-NPs & $150 \pm 10.7$ & $57.1 \pm 1.6$ & $0.106 \pm 0.027$ & $0.71 \pm 0.06$ \\
LNPs & $195 \pm 27.2$ & $-1.8 \pm 1.0$ & $0.159 \pm 0.072$ & $0.52 \pm 0.10$ \\
\hline
\end{tabular}

Abbreviations: ZP, zeta potential; PDI, polydispersity index; LC, loading content; PLGA-NPs, doxorubicin-loaded PLGA nanoparticles; LNPs, lipid/polymer particle assemblies. may improve the biocompatibility and facilitate further application in vivo. ${ }^{33}$ The colloidal stability of PLGA NPs and LNPs was determined in serum-supplemented RPMI-1640 by dynamic-light scattering. Compared to PLGA NPs, the LNPs showed excellent stability in this biomimetic medium over a 7-day period.

\section{In vitro drug release}

Drug release in vitro was determined in PBS of different $\mathrm{pH}$ $(5.5,6.5$, and 7.4$)$ to estimate the potential drug release characteristics of the nanoparticles under different physiological conditions. The release profiles revealed that the release of DOX from LNPs-DOX (with lipid shell, LC 0.52\%) was accelerated at low pH. As shown in Figure 3, the LNPsDOX released its drug more readily at $\mathrm{pH} 5.5,6.5$, and 7.4. The LNPs with a lipid shell exhibited less than $20 \%$ DOX release after the first 6 hours at $\mathrm{pH} 7.4$ (blood $\mathrm{pH}$ level), and approximately $32 \%$ slow leakage within 72 hours. This controlled release from LNPs-DOX was similar to that from NPs-DOX (without a lipid shell; supporting information S2), and indicates that aggregation proceeds independent of the spatial structure of the particle core and the lipid shell, and that the DOX release was affected more by the DOX-PLGA conjugation. In the more acidic tumor microenvironment ( $\mathrm{pH}$ 6.5), and in endosomes/lysosomes ( $\mathrm{pH}$ 5.5), DOX release from these assemblies was more rapid, suggesting that DOX can be released sharply during intracellular trafficking, which would be helpful to bypass drug efflux.

\section{Determination of MDR on MCF-7/ADR cells}

Parental MCF-7 and resistant MCF-7/ADR cells were purchased from ICBC, and maintained at $37^{\circ} \mathrm{C}$ in $5 \% \mathrm{CO}_{2}$ in RPMI-1640 medium with a $1 \%$ penicillin-streptomycin solution. Preliminary experiments and the MTT assays here showed that MCF-7/ADR had significant MDR. MCF-7 and MCF-7/ADR cells were exposed to increasing doses of DOX for 24 hours. Cell viability and $50 \%$ viability $\left(\mathrm{IC}_{50}\right)$ were determined (supporting information, S3). The $\mathrm{IC}_{50}$ of MCF-7/ADR $(47.53 \pm 2.53 \mu \mathrm{g} / \mathrm{mL})$ was 228 -fold greater than that in MCF-7 cells $(0.221 \pm 0.045 \mu \mathrm{g} / \mathrm{mL})$. To further understand the drug resistance in MCF-7/ADR, P-gp protein expression was determined by flow cytometry. The results revealed that the greater drug resistance of MCF-7/ADR was accompanied by the higher expression of P-gp protein (Figure 4), suggesting that reversal of resistance to DOX in MCF-7/ADR should escape this protein-mediated efflux. 

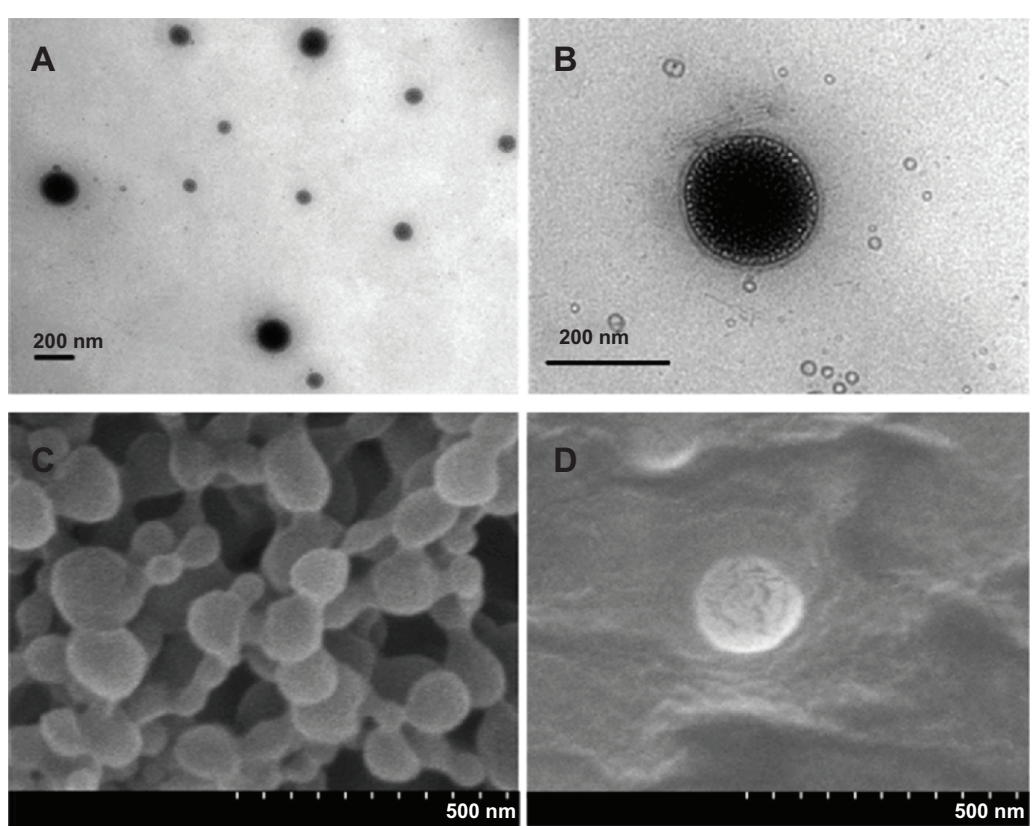

Figure 2 TEM and SEM images of LNPs formed with a core-shell supramolecular structure. A TEM image shows relatively uniform sized LNP particles (A), and a LipoParticle revealing its lipid shell thickness (B). A SEM image shows the fairly rigid surface morphology of PLGA particles by the electron-beam bombardment effect (C), and another SEM image displays the degradation and cracking of LNPs with a DPPC shell (D).

Abbreviations: DPPC, I,2-dipalmitoyl-sn-glycero-3-phosphocholine; LNPs, lipid/polymer particle assemblies; SEM, scanning electron microscopy; TEM, transmission electron microscopy.

\section{Intracellular accumulation of doxorubicin} DOX is known to interact with DNA by intercalation and inhibition of macromolecular biosynthesis. One potential strategy to reverse DOX resistance in cells is to change the intracellular localization of the drug through endocytosis using carrier particles. ${ }^{34}$ When drug-loaded nanocarriers enter cells via endocytosis, the particle-associated drugs can escape endosomal/lysosomal effect (eg, the proton sponge effect elicited by PEI) and increase the localization in the cytosol or nucleus of cells. ${ }^{35}$ Here, to determine if LNPsDOX could be accumulated efficiently in the nucleus, the intracellular localization of free DOX and LNPs-DOX was

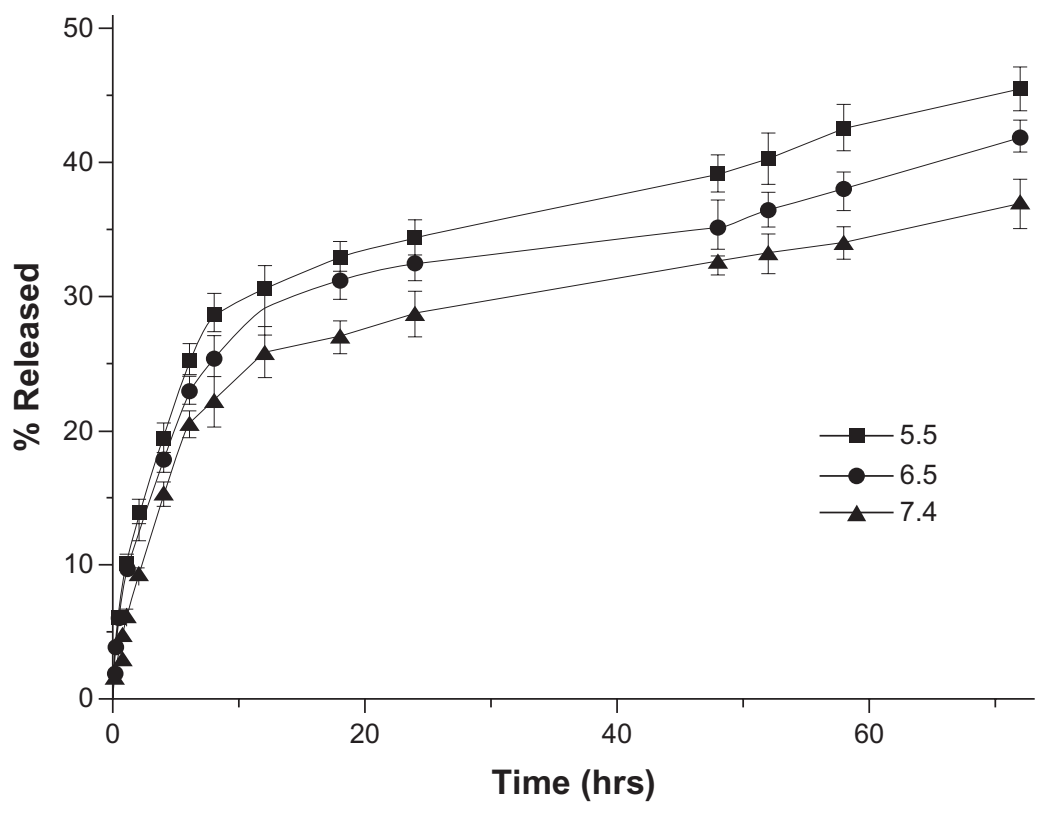

Figure 3 In vitro drug release profiles of DOX-loaded LNPs at different $\mathrm{pH}(5.5,6.5$, and 7.4$)$ and $37^{\circ} \mathrm{C}$. Abbreviations: DOX, doxorubicin; LNPs, lipid/polymer particle assemblies. 


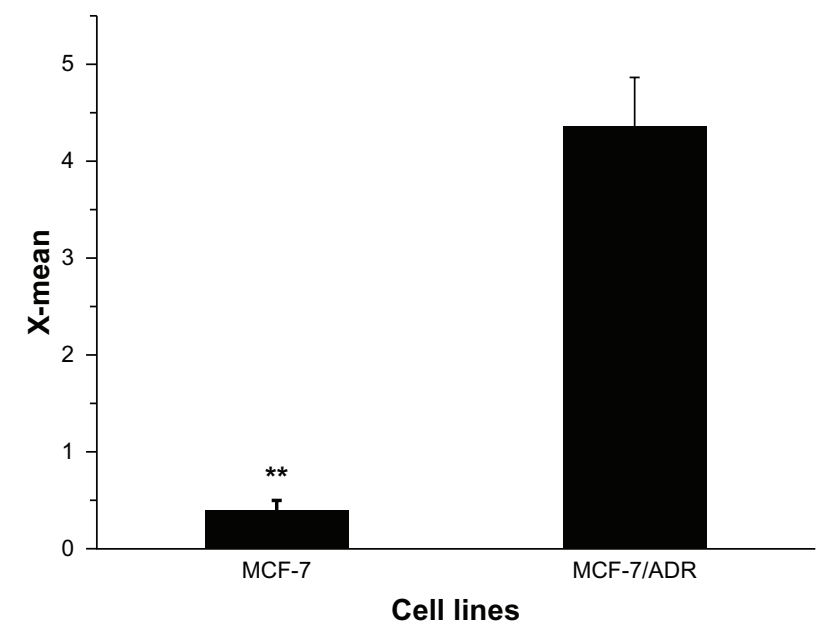

Figure 4 P-gp expression in MCF-7 and MCF-7/ADR cells, as shown by flow cytometry analysis.

Note: $* * p<0.01$

Abbreviations: MCF-7, Michigan Cancer Foundation-7; MCF-7/ADR, MCF-7/ adriamycin; P-gp, P-glycoprotein.

characterized in both parental MCF-7 and MCF-7/ADR cells. LNPs-DOX distribution in MCF-7 and MCF-7/ADR cells was visualized after 15 minutes, 1 hour, and 4-hour incubation (Figure 5). Following treatment in MCF-7/ADR cells, little free DOX could diffuse into the nucleus, and most of the drug accumulated in the cytoplasm ( 15 minutes -1 hour). Then, the drug uptake and accumulation in MCF-7/ADR cells was reduced significantly, which should be the result of the efflux of DOX caused by the overexpression of the ATP-binding cassette (ABC) transporters (Figure 5A). By contrast, the intracellular localization of LNPs-DOX in MCF-7/ADR cells was changed. After a 1-hour exposure, significant DOX was accumulated in the cytoplasm of the parental MCF-7 cells, whereas substantially increased LNPsDOX accumulated in the nuclei of the drug-resistant MCF-7/ ADR cells (Figure 5B). This observation suggests that LNPs, as a drug carrier, could change the sub-cellular localization of DOX and bypass MDR. The results at 4 hours showed the long-term accumulation of LNPs-DOX in the nuclei.

To further characterize the intracellular accumulation of DOX, the study determined the DOX concentration in MCF-7 and MCF-7/ADR cells (Figure 6). After treatment with free DOX (15 minutes drug exposure), the amount of drug that accumulated in MCF-7 cells was 2.6-fold greater than that in MCF-7/ADR cells. Following a 1-hour exposure to free DOX, the drug concentration in MCF-7/cells was about tenfold greater than that in MCF-7/ADR cells, the DOX within which were reduced due to active drugs efflux. In contrast to free DOX, the accumulation of LNPsDOX in MCF-7 and MCF-7/ADR cells were similar after
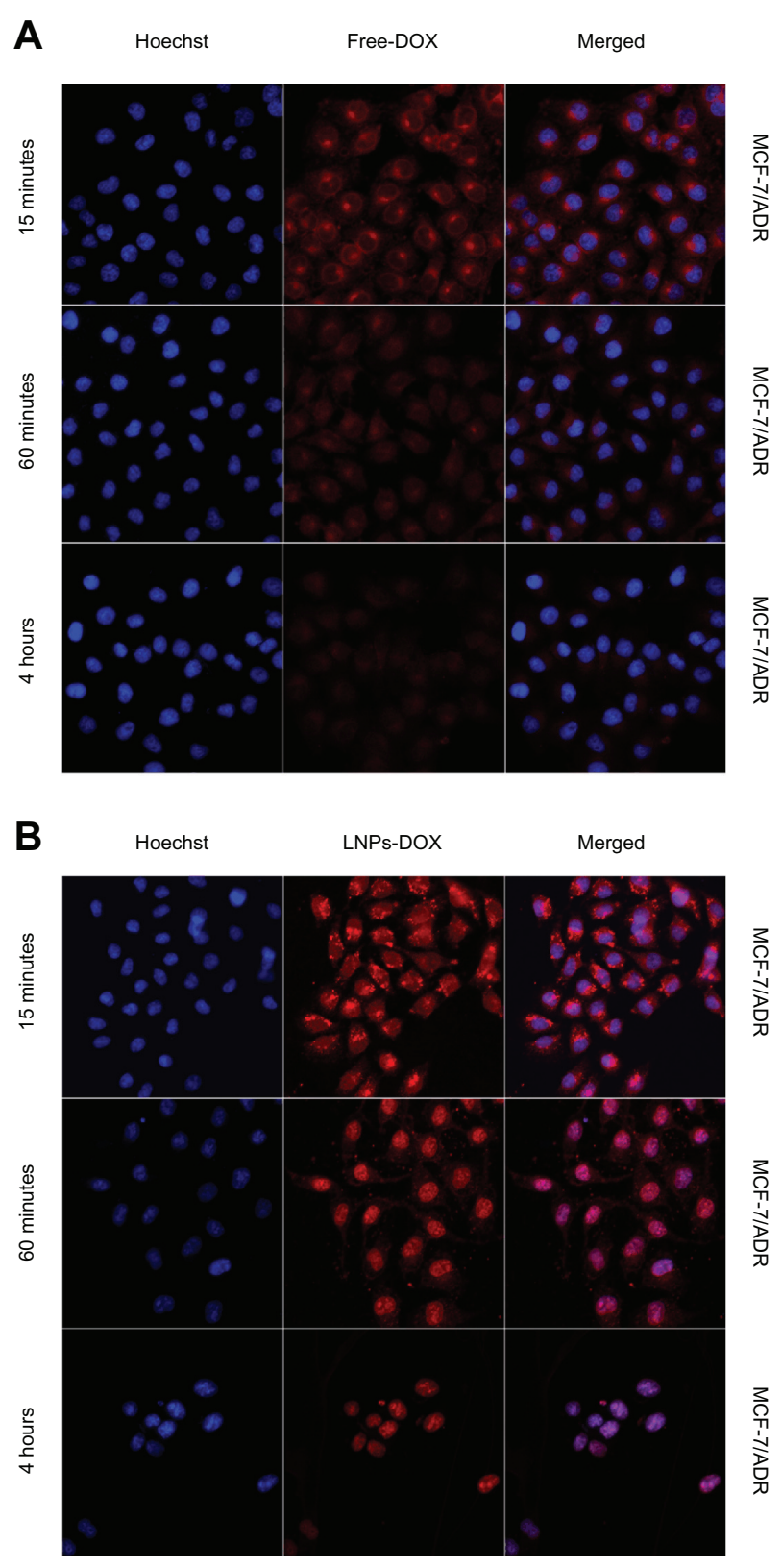

Figure 5 Confocal images of the intracellular accumulation of free DOX and LNPsDOX in MCF-7 (A) and MCF-7/ADR (B) cells. Cells were treated with $8 \mu \mathrm{g} / \mathrm{mL}$ free DOX or an equivalent concentration of LNPs-DOX for the different time periods as indicated. Nuclear (blue) uptake of DOX was increased dramatically by LNPs.

Abbreviations: DOX, doxorubicin; free DOX, free doxorubicin; LNPs-DOX, doxorubicin loaded in lipid/polymer particle assemblies; MCF-7, Michigan Cancer Foundation-7; MCF-7/ADR, MCF-7/adriamycin.

15 minutes drug exposure. After a 4-hour exposure, the LNPs-DOX concentration indicated no significant reduction in both cells. The phenomenon may demonstrate that the LNPs-DOX have successfully bypassed the enzymatic degradation and efflux pumps effect. More importantly, based on confocal laser scanning microscopy (Figure 5B), most of the DOX was located in the nuclei of the cancer cells at this time point. Since DOX is a drug that is toxic to the cell cycle 


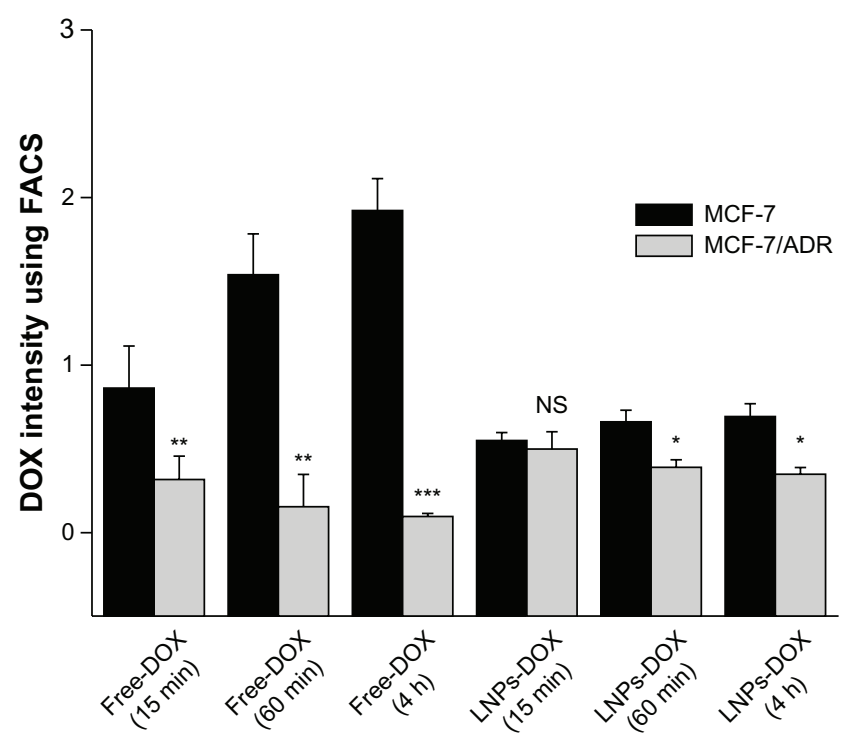

Figure 6 Accumulation of free DOX or LNPs-DOX in MCF-7 and MCF-7/ADR cells. Cells were treated with $8 \mu \mathrm{g} / \mathrm{mL}$ DOX or an equivalent concentration of LNPs-DOX for 15 minutes and I hour. The intracellular DOX was then determined by flow cytometry analysis.

Notes: $* P<0.05$; ** $P<0.01$; *** $p<0.001$.

Abbreviations: DOX, doxorubicin; free DOX, free doxorubicin; h, hours; LNPsDOX, doxorubicin loaded in lipid/polymer particle assemblies; MCF-7, Michigan Cancer Foundation-7; MCF-7/ADR, MCF-7/adriamycin; NS, no significance.

by inhibiting the activity of topoisomerase II, the cytotoxicity of LNPs-DOX would be increased.

\section{Reversal of MDR in vitro by LNPs}

Following the analysis of the intracellular accumulation of LNPs-DOX, the ability of LNPs to overcome MDR was investigated. The study evaluated the cytotoxicity in vitro of
DOX-loaded LNPs, blank LNPs, and free DOX to parental and resistant MCF-7 cells, using the MTT assay. The blank LNPs showed no cytotoxicity to either cell line; the viability values of MCF-7 and MCF-7/ADR cells were $98 \%$ and $97 \%$, respectively, at the blank LNPs concentration of $50 \mathrm{mg} / \mathrm{mL}$ (Figure 7). The LNPs-DOX and free DOX both exhibited significant cell growth inhibition of the parental cell line. The DOX-loaded LNPs exhibited a dose-dependent cytotoxicity against MCF-7/ADR cells (IC50:1.61 $\pm 0.13 \mu \mathrm{g} / \mathrm{ml}$ ). That is, DOX-loaded LNPs had a 30 -fold lower $\mathrm{IC}_{50}$ value than free DOX in MCF-7/ADR, suggesting that LNPs can circumvent drug efflux-mediated MDR.

\section{Discussion}

Adsorption of lipids onto oppositely charged surfaces or particles has been studied in detail by analytical and simulation methods. ${ }^{36}$ The study employed lipid/particle assemblies composed of positively charged PLGA nanoparticles (150 nm in diameter), covered by a bilayer of DPPC (200 nm for LNPs). In this context, DMAB consists of a bromide headgroup with two hydrophobic tails connecting them together; that is, one hydrophobic chain of DMAB is adsorbed onto the surface of PLGA nanoparticles; and the other acts as electrostatic glue that enhances the stability of the self-assembled particles by molecular electronegativity interaction with the oppositely charged phosphocholine headgroups. ${ }^{25}$ Once these cell-like structures were formed, the adsorption of a lipid bilayer on the PLGA core could be seen by TEM. Based on SEM, the surface of the particles

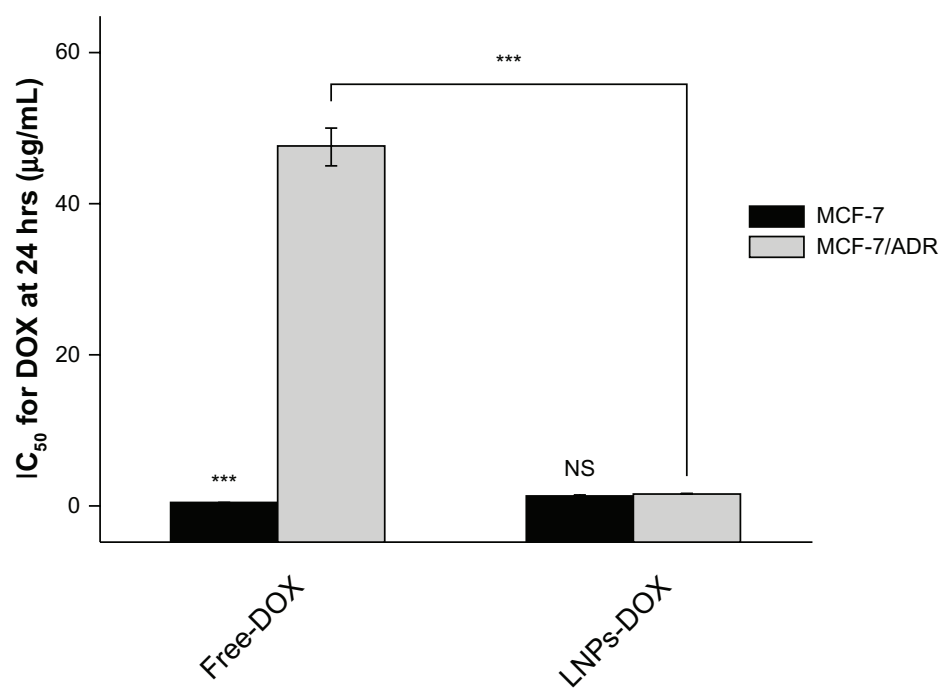

Figure $7 \mathrm{IC}_{50}$ for free DOX or LNPs-DOX in MCF-7 and MCF-7/ADR cells. Cells were treated with various concentrations of DOX or LNPs-DOX at the same dose for 24 hours. Note: $* * * p<0.001$.

Abbreviations: Free DOX, free doxorubicin; $\mathrm{IC}_{50}$, half maximal inhibitory concentration; h, hours; LNPs-DOX, doxorubicin loaded in lipid/polymer particle assemblies; MCF-7, Michigan Cancer Foundation-7; MCF-7/ADR, MCF-7/adriamycin; NS, no significance. 
was covered with DPPC, which constituted a biocompatible membrane environment useful for in vivo biomedicine (eg, in the delivery of drugs, proteins, and peptides).

LipoParticles are self-assemblies with a particle core surrounded by a lipid shell. PLGA particles have certain cell cytotoxicity by generating transient holes on cell membranes with a positively charged ammonium bromide headgroup of the DMAB molecule; ${ }^{37}$ however, the negative charges on the hydrophilic segment of DPPC could be attracted to the oppositely charged ions of the ammonium bromide ammonia headgroup. ${ }^{38}$ This charge neutralization would then form the lipid/particle assemblies, and improve the biocompatibility of the particles, with a reduction of the zeta potential from $57 \mathrm{mV}$ to $-1.8 \mathrm{mV}$. The MTT assays also confirmed that the blank LNPs at $50 \mathrm{mg} / \mathrm{mL}$ were not cytotoxic, and cell membrane damage caused by DMAB was not found. Thus, the modification of the cationic polymer substance with a lipid shell should be a useful means to reduce the toxicity of positively charged biomaterials, and there is potential to expand the application of these materials in biomedicine.

The MDR of MCF-7/ADR was accompanied with overexpression of $\mathrm{P}$-gp transporter. Bypassing these drug effluxes is a rational strategy to circumvent the MDR. ${ }^{39}$ In the current study, the significant cytotoxicity of LNPs-DOX in the MCF-7/ADR has been validated. Overcoming the MDR was related to the enhanced intracellular accumulation and nuclear targeting of the drug mediated by LNPs. To elaborate the details of the MDR reversal, the intracellular accumulation and localization of free DOX and LNPs-DOX was investigated in the drug-resistant MCF-7/ADR cells. Since the intracellular trafficking pathways of drug-loaded carriers, such as polymer capsules, were previously reported, ${ }^{10}$ the cellular uptake and fluorescence imaging studies were performed to determine how the newly designed lipid/ particle assemblies were able to bypass the resistance to DOX. The results showed that LNPs-DOX could effectively increase the DOX concentration in MCF-7/ADR cells, and most of the LNPs-DOX located in the nuclei of the drugresistant cells after about 1 hour of incubation. This observation demonstrated that the DMAB-modified particle core had the ability to escape from endosomes/lysosomes and localize in the nucleus. In summary, when the drug delivery system entered cells via endocytosis, after enzymatic degradation, the encapsulation of DMAB-modified particle core may be exposed to the endosomal/lysosomal lumen, and lead to the rupture of the particles. ${ }^{17}$ Then, the positively charged core would deliver the encapsulated DOX to the nucleus, which could increase DOX localization in the nucleus, and thereby bypass the drug efflux mechanism mediated by transporter proteins. The lipid/particle assemblies developed here could produce intracellular targeting to the nucleus and overcome the resistance of the cancer cells to DOX.

\section{Conclusion}

In conclusion, it can be reported that the lipid/particle assemblies consisting in DMAB-modified PLGA NPs coated with DPPC lipid shell have been successfully developed for MDR reversal. The results demonstrate that these LNPs, with biomimetic properties of lipids, can improve the biocompatibility of cationic particles via charge neutralization. Importantly, the LNPs can reverse the transporter-mediated drug resistance by increasing the intracellular accumulation and improving the targeting to the nucleus. This drug delivery system has the potential for overcoming MDR in biomedicine.

\section{Acknowledgments}

Support from the National Natural Science Foundation of China (NSFC: 30973647) and National Basic Research Program of China (973 Program, 2009 CB930300) are acknowledged.

\section{Disclosure}

The authors report no conflicts of interest in this work.

\section{References}

1. Szakács G, Paterson JK, Ludwig JA, et al. Targeting multidrug resistance in cancer. Nat Rev Drug Discov. 2006;5(3):219-234.

2. Fojo AT, Ueda K, Slamon DJ, et al. Expression of a multidrugresistance gene in human tumors and tissues. Proc Natl Acad Sci USA. 1987;84(1):265-269.

3. Kohno K, Sato S, Takano H, et al. The direct activation of human multidrug resistance gene (MDR1) by anticancer agents. Biochem Biophys Res Commun. 1989;165(3):1415-1421.

4. Ambudkar SV, Kimchi-Sarfaty C, Sauna ZE, et al. P-glycoprotein: from genomics to mechanism. Oncogene. 2003;22(47):7468-7485.

5. Fletcher JI, Haber M, Henderson MJ, et al. ABC transporters in cancer: more than just drug efflux pumps. Nat Rev Cancer. 2010;10(2):147-156.

6. Ren F, Chen R, Wang Y, et al. Paclitaxel-loaded poly(n-butylcyanoacrylate) nanoparticle delivery system to overcome multidrug resistance in ovarian cancer. Pharm Res. 2011;28(4):897-906.

7. Sharma AK, Zhang L, Li S, et al. Prevention of MDR development in leukemia cells by micelle-forming polymeric surfactant. J Control Release. 2008;131(3):220-227.

8. Lee ES, Na K, Bae YH. Doxorubicin loaded $\mathrm{pH}$-sensitive polymeric micelles for reversal of resistant MCF-7 tumor. $J$ Control Release. 2005; 103(2):405-418.

9. Patel NR, Rathi A, Mongayt D, et al. Reversal of multidrug resistance by co-delivery of tariquidar (XR9576) and paclitaxel using long-circulating liposomes. Int J Pharm. 2011;416(1):296-299.

10. Yan Y, Ochs CJ, Such GK, et al. Bypassing multidrug resistance in cancer cells with biodegradable polymer capsules. Adv Mater. 2010;22(47):5398-5403. 
11. Kievit FM, Wang FY, Fang C, et al. Doxorubicin loaded iron oxide nanoparticles overcome multidrug resistance in cancer in vitro. J Control Release. 2011;152(1):76-83.

12. Varkouhi AK, Scholte M, Storm G, et al. Endosomal escape pathways for delivery of biologicals. J Control Release. 2011;151(3):220-228.

13. Boussif O, Lezoualch F, Zanta MA, et al. A versatile vector for gene and oligonucleotide transfer into cells in culture and in vivo: polyethylenimine. Proc Natl Acad Sci U S A. 1995;92(16):7297-7301.

14. Lin C, Zhong Z, Lok MC, et al. Novel bioreducible poly(amido amine)s for highly efficient gene delivery. Bioconjug Chem. 2007;18(1):138-145.

15. Hariharan S, Bhardwaj V, Bala I, et al. Design of estradiol loaded PLGA nanoparticulate formulations: a potential oral delivery system for hormone therapy. Pharm Res. 2006;23(1):184-195.

16. Sonaje K, Italia JL, Sharma G, et al. Development of biodegradable nanoparticles for oral delivery of ellagic acid and evaluation of their antioxidant efficacy against cyclosporine A-induced nephrotoxicity in rats. Pharm Res. 2007;24(5):899-908.

17. Fay F, Quinn DJ, Gilmore BF, et al. Gene delivery using dimethyldidodecylammonium bromide-coated PLGA nanoparticles. Biomaterials. 2010;31(14):4214-4222.

18. Cirman T, Oresić K, Mazovec GD, et al. Selective disruption of lysosomes in HeLa cells triggers apoptosis mediated by cleavage of Bid by multiple papain-like lysosomal cathepsins. J Bio Chem. 2004;279(5):3578-3587.

19. Mei L, Sun H, Song C. Local delivery of modified paclitaxel-loaded poly(epsilon-caprolactone)/pluronic F68 nanoparticles for long-term inhibition of hyperplasia. J Pharm Sci. 2009;98(6):2040-2050.

20. Sahana DK, Mittal G, Bhardwaj V, et al. PLGA nanoparticles for oral delivery of hydrophobic drugs: influence of organic solvent on nanoparticle formation and release behavior in vitro and in vivo using estradiol as a model drug. J Pharm Sci. 2008;97(4):1530-1542.

21. Troutier AL, Ladavière C. An overview of lipid membrane supported by colloidal particles. Adv Colloid Interface Sci. 2007;133(1):1-21.

22. Sengupta S, Eavarone D, Capila I, et al. Temporal targeting of tumour cells and neovasculature with a nanoscale delivery system. Nature. 2005;436(7050):568-572.

23. Carmona-Ribeiro AM. Interactions between cationic liposomes and drugs or biomolecules. An Acad Bras Cienc. 2000;72(1):39-43.

24. Campanhã MT, Mamizuka EM, Carmona-Ribeiro AM. Interactions between cationic liposomes and bacteria: the physical-chemistry of the bactericidal action. J Lipid Res. 1999;40(8):1495-1500.

25. Troutier AL, Véron L, Delair T, et al. New insights into self-organization of a model lipid mixture and quantification of its adsorption on spherical polymer particles. Langmuir. 2005;21(22):9901-9910.
26. Troutier AL, Delair T, Pichot C, et al. Physicochemical and interfacial investigation of lipid/polymer particle assemblies. Langmuir. 2005;21(4):1305-1313.

27. Zuzzi S, Cametti C, Onori G. Polyion-induced aggregation of lipidic-coated solid polystyrene spheres: the many facets of complex formation in low-density colloidal suspensions. Langmuir. 2008;24(12):6044-6049.

28. Debin A, Kravtzoff R, Santiago JV, et al. Intranasal immunization with recombinant antigens associated with new cationic particles induces strong mucosal as well as systemic antibody and CTL responses. Vaccine. 2002;20(21-22):2752-2763.

29. Berton M, Sixou S, Kravtzoff R, et al. Improved oligonucleotide uptake and stability by a new drug carrier, the SupraMolecular Bio Vector (SMBV). Biochim Biophys Acta. 1997;1355(1):7-19.

30. Thevenot J, Troutier AL, Putaux JL, et al. Effect of the polymer nature on the structural organization of lipid/polymer particle assemblies. J Phys Chem B. 2008;112(44):13812-13822.

31. Yoo HS, Oh JE, Lee KH, et al. Biodegradable nanoparticles containing doxorubicin-PLGA conjugate for sustained release. Pharma Res. 1999;16(7):1114-1118.

32. Jun YJ, Park MK, Jadhav VB, et al. Tripodal amphiphiles tunable for self-assembly to polymersomes. J Control Release. 2010;142(1):132-137.

33. He P, Urban MW. Phospholipid-stabilized Au-nanoparticles. Biomacromolecules. 2005;6(3):1224-1225.

34. Jabr-Milane LS, van Vlerken LE, Yadav S, et al. Multi-functional nanocarriers to overcome tumor drug resistance. Cancer Treat Rev. 2008;34(7):592-602.

35. Shieh MJ, Hsu CY, Huang LY, et al. Reversal of doxorubicin-resistance by multifunctional nanoparticles in MCF-7/ADR cells. J Control Release. 2011;152(3):418-425.

36. Salvati A, Ristori S, Oberdisse J, et al. Small angle scattering and zeta potential of liposomes loaded with octa(carboranyl)porphyrazine. J Phys Chem B. 2007;111(35):10357-10364.

37. Verma A, Uzun O, Hu Y, et al. Surface-structure-regulated cellmembrane penetration by monolayer-protected nanoparticles. Nat Mater. 2008;7(7):588-595.

38. Wang B, Zhang L, Bae SC, et al. Nanoparticle-induced surface reconstruction of phospholipid membranes. Proc Natl Acad Sci USA. 2008;105(47):18171-18175.

39. Kopecka J, Campia I, Olivero P, et al. A LDL-masked liposomaldoxorubicin reverses drug resistance in human cancer cells. J Control Release. 2011;149(2):196-205. 


\section{Supplementary Data}

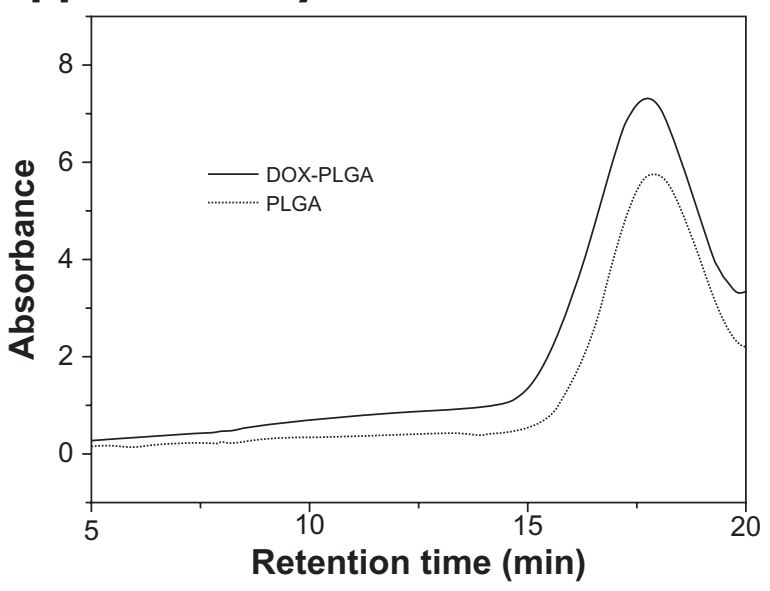

Figure SI Gel Permeation chromatogram of DOX-PLGA conjugate (solid line) and unconjugated PLGA (dotted line)

Abbreviations: DOX, doxorubicin; PLGA, poly(lactic-co-glycolic) acid.

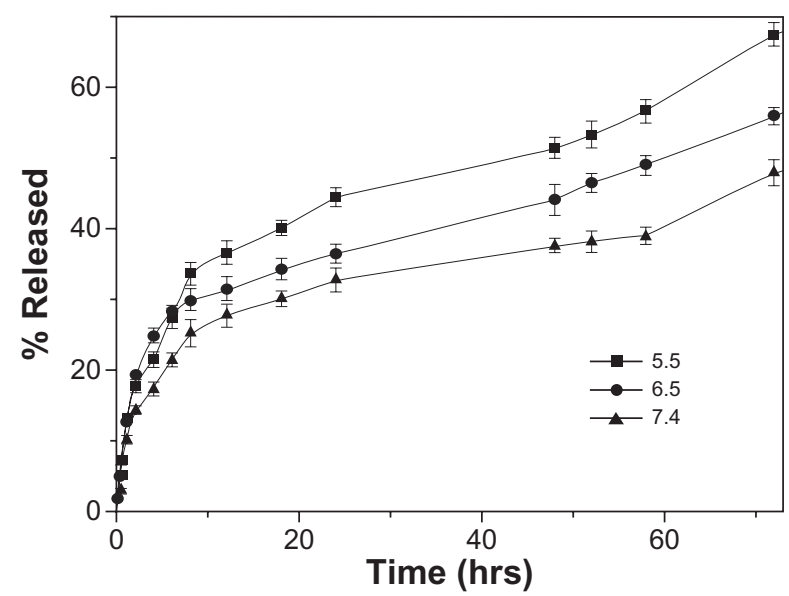

Figure S2 In vitro drug release profiles of DOX-loaded PLGA nanoparticles (without DPPC shell) in different $\mathrm{pH}(5.5,6.5$, and 7.4$)$ at $37^{\circ} \mathrm{C}$. Error bars indicate mean \pm SD. Abbreviations:DOX, doxorubicin;DPPC, I,2-dipalmitoyl-sn-glycero-3-phosphocholine; PLGA, poly(lactic-co-glycolic) acid; SD, standard deviation.

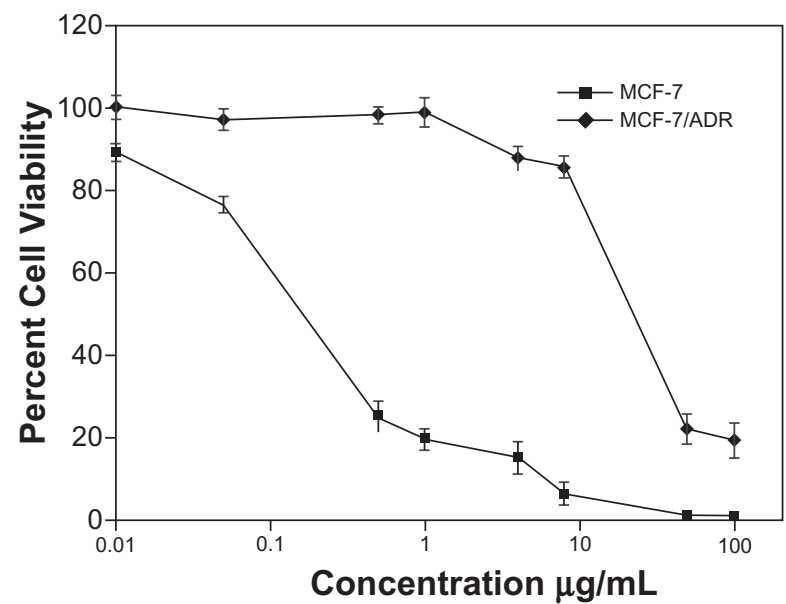

Figure S3 Cytotoxicity of Free-DOX against MCF-7 and MCF-7/ADR cells. Error bars indicate mean \pm SD.

Abbreviations: DOX, doxorubicin; MCF-7, Michigan Cancer Foundation-7; MCF-7/ ADR, MCF-7/adriamycin; SD, standard deviation.
International Journal of Nanomedicine

\section{Publish your work in this journal}

The International Journal of Nanomedicine is an international, peerreviewed journal focusing on the application of nanotechnology in diagnostics, therapeutics, and drug delivery systems throughou the biomedical field. This journal is indexed on PubMed Central, MedLine, CAS, SciSearch ${ }^{\circledR}$, Current Contents ${ }^{\circledR} /$ Clinical Medicine,

\section{Dovepress}

Journal Citation Reports/Science Edition, EMBase, Scopus and the Elsevier Bibliographic databases. The manuscript management system is completely online and includes a very quick and fair peer-review system, which is all easy to use. Visit http://www.dovepress.com/ testimonials.php to read real quotes from published authors 